\title{
User involvement in care planning
}

\section{The Care Programme Approach (CPA) from the users' perspective}

\author{
Mike Lawson, Chrissie Strickland and Paul Wolfson
}

\begin{abstract}
Alms and method To assess the extent of user involvement in care planning, from the users' perspective. Fiffy Care Programme Approach (CPA) meetings across five consultant teams were Included using quota sampling. Data concerning user involvement was collected by direct observation, semi-structured interviews with users and keyworkers and a review of CPA documentation.

Results User involvement in needs assessment and decision-making was poor, as was knowledge of care planning and information provision to users.

Clinical implications Limited resources and conflict resulting from the more containing aspects of the CPA remain obstacles for user involvement. Possible improvements include user led needs assessment, information pack provision and limiting attendance at CPA meetings.
\end{abstract}

The original guidelines (Department of Health, 1990) for implementation of the Care Programme Approach (CPA) stress the importance of user involvement in care planning. Features of the CPA which encourage this include a keyworker. multi-disciplinary working, regular reviews and ownership of a care plan which the user has signed.

Our aim was to evaluate the degree of user involvement in the CPA in a London borough, using discharge and review meetings as a central focus, as evidence suggests users may find these meetings unhelpful (Carson \& Sharma, 1994).

\section{The study}

The researcher was notified of all reviews and discharge meetings from January 1997. This continued until 10 data sets (five review and five discharge meetings) were completed for each of the five consultant teams at Bexley Hospital, providing a cross sectional quota sample $(n=50)$. Inclusion criteria were complex levels of the CPA. age range 18-75 years, and for discharge meetings, a minimum length of stay in hospital of 20 days.
Verbal consent was obtained from the user prior to the meeting. The researcher explained he was collecting information about how involved the user was in the planning of their care. in order to see how services could be improved. The researcher emphasised that he was not part of the clinical team, and any information collected for the purposes of the study would be confidential. Users were informed they were free to withdraw consent at any time.

Keyworker interviews collected clinical information and a Clinical Global Impression, Severity of Illness rating (CGI-S; Guy, 1976). Socio-demographic data were obtained from case notes. User involvement in meetings was assessed firstly by direct observation, to determine the number of people attending and what percentage of the meeting the user was present. Immediately afterwards, a semi-structured interview with the user assessed understanding of care planning, whether they felt their goals were discussed and considered adequately, whether they had seen a copy of their care plan and what their views were on how the service was provided. Finally, an open question invited comments on involvement in their care.

\section{Findings}

Fifty $(61 \%)$ of 82 identified users were interviewed. Five $(10 \%)$ users interviewed were detained under the Mental Health Act in hospital. The mean age of the group was 41 years (range 18-74). Ethnicity of users interviewed $(n=50)$ was: White $(n=45)$, Black African $(n=2)$, Black Caribbean $(n=1)$, Indian $(n=1)$ and Moroccan $(n=1)$. Forty-eight $(96 \%)$ had previous psychiatric admissions - $34(68 \%)$ having been admitted four or more times. The mean CGI-S score was 2.72 , on a scale of 1-7, between 'borderline' and 'mildly' ill. Case note diagnosis using ICD-10 (World Health Organization, 1992) was: schizophrenia or related disorder $27(54 \%)$ : affective disorders 16 (32\%); neurotic/stress- 
related disorders three $(6 \%)$; personality disorders four $(8 \%)$. Eighteen $(36 \%)$ of the users interviewed had a secondary diagnosis (alcohol/substance misuse, learning disabilities, physical disabilities).

A further 32 users could not be included in the study because staff felt it would be too distressing for the user if a researcher attended the meeting, the user did not give consent or the user did not attend the meeting. The non-study group were significantly more likely to have a history of 'drug or alcohol misuse' or secondary 'physical disabilities'. A diagnosis of schizophrenia appears more likely in the non-study group, although this fell short of significance (see Table 1).

The mean number of people at each meeting, excluding the researcher, was six (range 3-12), and the mean percentage of time a user was present during the meeting was $81 \%$ (range 29 $100)$. Thirty-three $(66 \%)$ users felt their goals had been fully discussed at the meeting, $10(20 \%)$ partially and seven (14\%) not at all. A care plan was produced in four review meetings $(8 \%)$. Nineteen (38\%) users demonstrated an adequate understanding of what a care plan meant (guideline: "an individual plan detailing aspects of the users care"). Following explanation where necessary, $32(64 \%)$ users said they would like a copy of their care plan and $23(46 \%)$ were prepared to sign it immediately, while a further $13(26 \%)$ would consider signing after they had seen it. Fourteen (28\%) said they would not sign their care plan. Four $(8 \%)$ said they had received a copy and been asked to sign it.

Thirty-six (72\%) users wanted more information on medication and side-effects, $34(68 \%)$ on diagnosis and $31(62 \%)$ on services available in the community. Forty $(80 \%)$ users were unaware of the advocacy service. Twenty-five (50\%) users were unaware of who would be attending the meeting. Forty-six (92\%) users were satisfied with their keyworker.

Thirty-nine (78\%) users chose to make general comments which were grouped subsequently by the authors. Those comments reported more than once were: happy with their care (19), too many people at meetings (19), not consulted or listened to (seven), general dissatisfaction with care (four), threats of legal action (two) and suggestions to improve involvement (two). Eleven (22\%) users' notes contained a current care plan, four $(8 \%)$ of which contained user-led goals. There was no evidence of any formal needs assessment taking place.

\section{Discussion}

Everyone in the study group had a keyworker, knew who they were, and nearly all were satisfied with this arrangement. The majority of users wanted to be more involved in their care and wanted to own a copy of their care plan. Implementation was poor in this area. The continuing need for updated information about medication, diagnosis and available community services was not reflected either in care planning or the content of the meetings. The local branch of MIND has now produced an information pack. co-written by users, in an attempt to redress the situation.

CPA meetings are designed to reduce communication fatlures and facilitate user involvement. Simplifying the related paper work may free keyworkers' time for other duties, such as a formal user-assisted self-assessment (Avon Mental Health Measure Working Group, 1996). This would allow the user's goals to be identified and included in care planning more consistently.

Promoting the rights of individuals with mental health problems may come into conflict with the coercive and containing powers available to mental health services. Users may be acutely aware of this. They may also find it unfair to be expected to talk about personal problems in front of a large group of people. One suggestion could be for the care coordinator to negotiate with all concerned prior to the meeting. This would change the purpose of the meeting to a formal

Table 1. Differences between the study group and non-study group $\left(\chi^{2}\right)$

\begin{tabular}{lccc}
\hline Variable & $\begin{array}{c}\text { Study group frequency } \\
(\mathbf{n = 5 0 )}\end{array}$ & $\begin{array}{c}\text { Non-study group frequency } \\
(\boldsymbol{n}=\mathbf{3 2 )}\end{array}$ & $\begin{array}{c}\text { Two-tailed } \\
\text { P value }\end{array}$ \\
\hline Ethnicity White) & 45 & 30 & 0.55 \\
Living with partner & 22 & 12 & 0.56 \\
Mental Health Act & 5 & 2 & 0.55 \\
Supervision Register & 10 & 6 & 0.89 \\
Previous admissions & 48 & 31 & 0.84 \\
Diagnosis of schizophrenia & 27 & 24 & 0.06 \\
History of drug or alcohol misuse & 3 & 10 & $0.002^{*}$ \\
Memory/concentration deficits & 17 & 8 & 0.39 \\
Physical disabilities & 3 & 10 & $0.002^{*}$ \\
\hline
\end{tabular}

${ }^{*} P<0.005$ 
ratification, but would be a complex and time consuming process for the care coordinator.

User involvement is a difficult concept to quantify, although users were involved in devising the key indicators used. There is clearly a need for the development of valid and reliable measures of user involvement. This study is also limited by its relatively small sample. Due to the significant differences found between the study and non-study group, the $61 \%$ of users who participated are unlikely to be representative. There is a known association between substance misuse, which is significantly more prevalent in the non study group, and poor engagement with services (Sparr et al, 1993). Those who did not attend review meetings may feel less involved with their care. Physical disabilities, which were significantly more common in the non-study group, may have contributed to non-attendance. The focus of the study on the aftermath of CPA meetings had the advantage of immediacy and good recall, but may not have allowed users time to reflect on what happened during the meeting.

\section{Acknowledgements}

We thank S. Garrett, P. Skarratt, S. Norman, J. Saunders and R. Echlin for their help and support.

\section{References}

avon Mental health Measure Working Group (1996) The Avon Mental Health Measure: A User-Centred Approach of Assessing Need. Bristol: Bristol Social Services.

CaRSON, J. \& SHARMA. T. (1994) In-patient psychlatric care: What helps? Staff and patient perspectives. Joumal of Mental Health (UK, 3, 99-104.

Department of HEALTH (1990) The Care Programme Approach for People with a Mental Illness Referred to Specialist Psychiatric Services. London: HMSO.

GuY, W. (1976) ECDEU Assessment Manual for Psychopharmacology. Revised DHEW pub. no. (ADM) 78-388. Rockville, MD: National Institute of Mental Health.

SPARR, L. F., MOFFTt, M. C. \& WARD, M. F. (1993) Missed psychlatric appointments: who returns and who stays away. American Journal of Psychiatry. 150. 801-805.

WORLD HEALTH ORGanization (1992) The Tenth Revision of the International Classification of Diseases and Related Health Problems. Geneva: WHO.

Michael Lawson, Assistant Psychologist; "Paul Wolfson, Consultant Psychiatrist, Oxleas NHS Trust, Bexley Hospital, Kent DA5 2BW: and Chrissie Strickland, Director of MIND, Bexley, Kent

*Correspondence

\title{
Audit of a recently introduced stimulus dosing policy in an electroconvulsive therapy clinic
}

\author{
G. Shaikh, R. Ireland, M. McBreen and R. Ramana
}

\begin{abstract}
Aims and method To audit the clinical practice of selzure threshold estimation and subsequent stimulus dose adjustment in the electroconvulsive therapy (ECT) clinic. Case notes of patients who had ECT over a sixmonth period were audited. Results were discussed at an audit meeting and guidelines and training modified appropriately prior to the second cycle of the audit. Reeults Initial dose titration was poor in the first period. but improved in the second. The majority of patients were insufficiently stimulated after missed selzures in both periods and stimulus doses were not being
\end{abstract}

reduced following prolonged seizures.

Clinical implications The audit identified the need for continuing supervision of trainees in addition to clear training and guidelines.

Electroconvulsive therapy (ECT) is a recognised and effective treatment for severe depression. A response to treatment requires a moderately supra-threshold stimulus. Doses marginally above the seizure threshold do not have a 